\title{
Theory of Enhanced Reversed Shear Mode in Tokamaks
}

\author{
K.C. Shaing, A.Y. Aydemir \\ Institute for Fusion Studies, The University of Texas at Austin, Austin, Texas 78712 USA
}

W.A. Houlberg

Fusion Energy Division, ORNL, P.O. Box 2009, Oak Ridge, TN 37831-8071 USA

M.C. Zarnstorff

Princeton Plasma Physics Laboratory, P.O. Box 451, Princeton, NJ 08543

(April 29, 1998)

\begin{abstract}
It is shown that toroidal magnetic field ripple induced particle flux can drive poloidal $\boldsymbol{E} \times \boldsymbol{B}$ speed to bifurcate over the local maximum of the nonlinear poloidal (or parallel) viscosity. Here, $\boldsymbol{E}$ is the electric field and $\boldsymbol{B}$ is the magnetic field. This mechansim, together with the turbulence suppression due to the radial gradient of the $\boldsymbol{E} \times \boldsymbol{B}$ and diamagnetic angular velocity, is employed to explain enhanced reversed shear mode observed in the core region of tokamaks.
\end{abstract}

$52.55 \mathrm{Fa}, 52.55 . \mathrm{Fi}$

Typeset using REVTEX 
Improved plasma confinement regime (H-mode) has been observed in the edge region of many tokamaks and stellarators [1]- [6]. It is initiated by a sudden increase in the magnitude of the poloidal $\boldsymbol{E} \times \boldsymbol{B}$ speed and followed by the turbulence suppression which leads to better plasma confinement $[2,4]$. Here $\boldsymbol{E}$ is the electric field and $\boldsymbol{B}$ is the magnetic field. A quantitative theory based on the bifurcation of the poloidal $\boldsymbol{E} \times \boldsymbol{B}$ Mach number $U_{p m}$ over the local maximum of the nonlinear plasma viscosity and the subsequent turbulence suppression due to the radial gradient of the $\boldsymbol{E} \times \boldsymbol{B}$ and diamagnetic angular velocity is in good agreement with both electrode induced and naturally occurred H-mode $[7,8]$. Here, we extend the H-mode theory to explain the improved plasma confinement regime - enhanced reversed shear (ERS) mode - in the core region $[9,10]$. Indeed, it is observed in TFTR that there is a sudden jump in radial electric field prior to the onset of ERS mode [11]. The corresponding value of $U_{p m}$ is greater than unity. This phenomenon is the same as that in H-mode. Furthermore, because the slope of the radial electric field changes sign in the bifurcation layer, the appropriate bifurcation quantity is the radial electric field and not the gradient of the radial electric field. Confinement improvement theory based on the bifurcation of $U_{p m}$ is consistent with this observation.

It is obvious that ion orbit loss mechanism which is responsible for the bifurcation of the poloidal $\boldsymbol{E} \times \boldsymbol{B}$ speed in naturally occurred H-mode is not effective in the core region because the number of particles that intersect plasma boundary is small. One therefore has to find a different mechanism to drive $U_{p m} \equiv V_{\| i} / v_{t i}-c E_{r} / v_{t i} B_{p}$ over the local maximum of the parallel (or poloidal) viscosity. Here, $V_{\| i}$ is the ion parallel flow, $c$ is the speed of light, $E_{r}$ is the radial electric field, $B_{p}$ is the poloidal magnetic field strength, and $v_{t i}$ is the ion thermal speed. There are such mechanisms. One possible mechanism is the momentum input associated with the injected neutral particle beam. The other is the toroidal magnetic field ripple induced particle flux. Because enhanced reversed shear mode exists even in the balanced neutral particle beam injection operations, we focus on the bifurcation of $U_{p m}$ driven by the ripple induced particle flux. Note that ripple trapped particle flux can drive bifurcation is already demonstrated in Refs. [12] and [13]. In Ref. [12], it is shown that 
electron $1 / \nu$ flux can cause radial electric field to bifurcate to a more positive value. In Ref. [13], ion $1 / \nu$ flux is used to drive radial electric field to bifurcation in the stellarator H-mode theory.

There are two types of ripple induced particle flux. One is due to ripple trapping [14]. In the relatively collisional regime, it contributes to the $1 / \nu$ flux [15]. The other is due to the modification of the trajectories of the toroidally trapped particles (i.e. bananas) and barely toroidally trapped or circulating particles by the magnetic field ripple. This class of orbits is not trapped in the ripple well. In the low collisionality regime, it contributes to the ripple plateau flux $[16,17]$. The constitutive relations between ripple induced particle flux and the components of the viscous tensor are adequately understood. We employ these constitutive relations in momentum equation.

The momentum equation is

$$
\sum_{a} n_{a} m_{a} \frac{d \boldsymbol{V}_{a}}{d t}=\frac{1}{c} \boldsymbol{J} \times \boldsymbol{B}-\boldsymbol{\nabla}\left(\sum_{a} p_{a}\right)-\boldsymbol{\nabla} \cdot\left(\sum_{a} \boldsymbol{\pi}_{a}\right)+\left(\sum_{a} \boldsymbol{S}_{m a}-\sum_{a} m_{a} \boldsymbol{V}_{a} S_{n a}\right)
$$

where the subscript $a$ indicates plasma species, $n_{a}$ is plasma density, $m_{a}$ is the mass, $\boldsymbol{V}_{a}$ is the flow velocity, $\boldsymbol{J}$ is plasma current, $p_{a}$ is plasma pressure, $\boldsymbol{\pi}_{a}$ is the viscous tensor, $\boldsymbol{S}_{m a}$ is the momentum source, and $S_{n a}$ is the particle source. Note that particle source associated with gas puffing or pellet injection reduces velocity. The $d / d t$ operator in Eq. (1) is $d / d t=\partial / \partial t+\boldsymbol{V}_{a} \cdot \boldsymbol{\nabla}$. The steady state parallel component of Eq. (1) is

$$
\left\langle\boldsymbol{B} \cdot \boldsymbol{\nabla} \cdot \sum_{a} \boldsymbol{\pi}_{a}\right\rangle=\left\langle\boldsymbol{B} \cdot \sum_{a} \boldsymbol{S}_{m a}\right\rangle-\left\langle\boldsymbol{B} \cdot \sum_{a} m_{a} \boldsymbol{V}_{a} S_{n a}\right\rangle,
$$

where the angular brackets denote flux surface averaging. Because we are interested in the case where $U_{p m} \sim 1$ but $\left|\boldsymbol{V}_{a}\right| / v_{t a}<1$, the convective term $\boldsymbol{V}_{a} \cdot \boldsymbol{\nabla} \boldsymbol{V}_{a}$ can be neglected. Here, $v_{t a}$ is the thermal speed of species $a$. The steady state toroidal component of Eq. (1) is

$$
\left\langle\boldsymbol{B}_{t} \cdot \nabla \cdot \sum_{a} \boldsymbol{\pi}_{a}\right\rangle=\left\langle\boldsymbol{B}_{t} \cdot \sum_{a} \boldsymbol{S}_{m a}\right\rangle-\left\langle\boldsymbol{B}_{t} \cdot \sum_{a} m_{a} \boldsymbol{V}_{a} S_{n a}\right\rangle .
$$

We employ here Hamada coordinates: $\boldsymbol{B}=\boldsymbol{B}_{t}+\boldsymbol{B}_{p}=\psi^{\prime} \nabla V \times \boldsymbol{\nabla} \theta-\chi^{\prime} \nabla V \times \boldsymbol{\nabla} \zeta$ where $\boldsymbol{B}_{t}$ is the toroidal magnetic field, $\boldsymbol{B}_{p}$ is the poloidal magnetic field, $\psi^{\prime}=\boldsymbol{B} \cdot \boldsymbol{\nabla} \zeta, \chi^{\prime}=\boldsymbol{B} \cdot \boldsymbol{\nabla} \theta$, 
$\theta$ is the poloidal angle, $\zeta$ is the toroidal angle, and $V$ is the volume enclosed in the flux surface [18]. Note that $\left\langle\boldsymbol{B}_{t} \cdot \boldsymbol{J} \times \boldsymbol{B}\right\rangle$ is related to $\partial\langle\boldsymbol{E} \cdot \boldsymbol{\nabla} V\rangle / \partial t$ through Amperé's law and vanishes at the steady state. Equations (2) and (3) completely determine the flow velocity $\boldsymbol{V}_{a}$ within the flux surface. To the leading order of gyroradius expansion $\boldsymbol{V}_{a}=V_{\| a} \widehat{n}+\boldsymbol{V}_{\perp a}$, and $\left.\boldsymbol{V}_{\perp a}=c \boldsymbol{B} \times \nabla \Phi / B^{2}+c \boldsymbol{B} \times \nabla p_{a} / n_{a} e_{a} B^{2}\right)$, where $\widehat{n}=\boldsymbol{B} / B, \quad \Phi$ is the electrostatic potential, and $e_{a}$ is the electric charge. Because $\Phi=\Phi(V)$ and $p_{a}=p_{a}(V), \boldsymbol{V}_{a} \cdot \nabla V=0$. Thus, there are two unknowns: parallel flow $V_{\| a}$ and $\boldsymbol{\nabla} \Phi \cdot \boldsymbol{\nabla} V$ to be determined from Eqs. (2) and (3). For simplicity, we only consider electron-ion plasmas. Because electron friction is much larger than electron viscous force in a large aspect ratio tokamaks, $V_{\| i} \approx V_{\| e}$ to the leading order of the ratio of electron viscous force to the electron friction force.

From the general expressions for the plasma viscosity in the plateau regime, we can evaluate ripple plateau viscosity for a model rippled tokamak $B=B_{0}(1-\epsilon \cos \theta-\delta \cos N \zeta)$ where $B_{0}$ is $B$ on the magnetic axis, $N$ is the number of toroidal magnetic field coils, $\epsilon$ is the inverse aspect ratio and $\delta$ is the ripple amplitude to obtain [19]

$$
\begin{aligned}
\left\langle\boldsymbol{B}_{t}\right. & \left.\cdot \boldsymbol{\nabla} \cdot \boldsymbol{\pi}_{a}\right\rangle_{r p}=\left\langle\boldsymbol{B} \cdot \boldsymbol{\nabla} \cdot \boldsymbol{\pi}_{a}\right\rangle_{r p}=\frac{\sqrt{\pi}}{4} n_{a} m_{a} \frac{v_{t a}^{2}}{R q} C_{1} B_{0}\left(N q \delta^{2}\right)\left(\frac{B_{p}^{2}}{B}\right)^{2}\left[\frac{V_{\| a}}{v_{t a}}\left(\frac{B}{B_{p}}\right)^{2}\right. \\
& \left.+\left(\frac{c E_{r}}{B_{p} v_{t a}}-\frac{c p_{a}^{\prime}}{n e B_{p} v_{t a}}\right)+\frac{1}{5}\left(\frac{q_{\| a}}{p_{a} v_{t a}}\left(\frac{B}{B_{p}}\right)^{2}-\frac{5}{2} \frac{c T_{a}^{\prime}}{e_{a} B_{p} v_{t a}}\right)\right]
\end{aligned}
$$

where prime denotes $d / d r, R$ is the major radius, $r$ is the local minor radius, $C_{1}=2$, $q$ is the safety factor, $q_{\| a}$ is the parallel heat flow, $T_{a}$ is the temperature, and subscript $r p$ indicates ripple plateau regime. We have approximated Hamada coordinates with cylindrical coordinates in Eq. (4). Note that electron ripple plateau viscosity is much smaller than that of ions and can be neglected. Because we are interested in the case $U_{p m} \sim 1$ and $B_{p} / B \ll 1$, we conclude ripple plateau viscosity mainly damps toroidal flow. Indeed, for $T_{i}=5 \mathrm{keV}$, $R=2.6 \mathrm{~m}, q=2.5, N=20$, and $\delta=1.3 \times 10^{-3}$, the toroidal momentum damping time $\tau_{M}$ is $\tau_{M}^{-1}=(\sqrt{\pi} / 2) C_{1}\left(v_{t i} / R q\right)\left(N q \delta^{2} / 2\right) \simeq 11.6 s^{-1}$ or $\tau_{M}=0.086 \mathrm{~s}$. Because this damping time is much shorter than the diffusive neoclassical toroidal viscosity [20], we neglect the latter contribution here. Note that the contribution from the $\epsilon \cos \theta$ variation of $B$ to $\langle\boldsymbol{B} \cdot \boldsymbol{\nabla} \cdot \boldsymbol{\pi}\rangle$ 
is ignored in Eq. (4) because we assume that toroidally trapped particles are in the banana regime which contributes to the nonlinear viscosity.

Ripple trapping induced particle flux has several collisionality regimes. Here, we only employ the $1 / \nu$ flux $\Gamma_{1 / \nu}$ calculated in Refs. [15] and [21] to demonstrate the fundamental bifurcation physics. The corresponding viscosity is [13]

$$
\begin{aligned}
\left\langle\boldsymbol{B}_{p}\right. & \left.\cdot \boldsymbol{\nabla} \cdot \boldsymbol{\pi}_{i}\right\rangle_{1 / \nu}=-\left\langle\boldsymbol{B}_{t} \cdot \boldsymbol{\nabla} \cdot \boldsymbol{\pi}_{i}\right\rangle_{1 / \nu}=-\frac{e}{c} B_{p} B \Gamma_{1 / \nu} \\
& =-\frac{64 G(\alpha)}{9(2 \pi)^{3 / 2}} n_{i} \epsilon^{2} \delta^{3 / 2}\left(\frac{c T_{i}}{e B r}\right)^{2} \frac{27.4}{\nu_{i}}\left[\left(\frac{p_{i}^{\prime}}{p_{i}}-\frac{e E_{r}}{T_{i}}\right)+2.37 \frac{T_{i}^{\prime}}{T_{i}}\right]\left(\frac{e B B_{p}}{c}\right),
\end{aligned}
$$

where $\alpha=\epsilon /(N q \delta)$, and $\nu_{i}$ is ion-ion-collision frequency. Note that $G(\alpha)$ is a function that accounts for the variation of the local ripple well depth, and for $\alpha \gg 1, G(\alpha) \simeq 0.02 / \alpha^{3}$ [15]. It is obvious from Eq. (5) $\left\langle\boldsymbol{B} \cdot \boldsymbol{\nabla} \cdot \boldsymbol{\pi}_{i}\right\rangle_{1 / \nu}=0$. Because electron $1 / \nu$ flux is smaller than that of ions, it is neglected.

The nonlinear resonant plasma viscosity due to banana and circulating particles is $[22,23]$

$$
\langle\boldsymbol{B} \cdot \boldsymbol{\nabla} \cdot \boldsymbol{\pi}\rangle_{n}=n_{a} m_{a} B^{2}\left(\mu_{1 a} U_{\theta a}+\frac{2}{5} \mu_{2 a} \frac{q_{\theta a}}{p_{a}}\right)
$$

where the subscript $n$ indicates nonlinear viscosity, $U_{\theta a}=\left(v_{t a} / B\right)\left[V_{\| a} / v_{t a}-c E_{r} /\left(B_{p} v_{t a}\right)+\right.$ $\left.c p_{a}^{\prime} /\left(n_{a} e_{a} v_{t a} B_{p}\right)\right]$, and $q_{\theta a} / p_{a}=\left(v_{t a} / B\right)\left[q_{\| a} /\left(p_{a} v_{t a}\right)+(5 / 2) c T_{a}^{\prime} /\left(e_{a} v_{t a} B_{p}\right)\right]$. Because $V_{\| e} \approx$ $V_{\| i} \ll v_{t e}$, and $c E_{r} /\left(B_{p} v_{t e}\right) \ll 1$, electron viscosity is not affected in the regime where $U_{p m} \sim 1$, standard electron viscosity coefficients [24] apply. The ion nonlinear resonant viscosity coefficients are $[22,23]\left(\mu_{1 i}, \mu_{2 i}\right)=\int_{\left|U_{p m}\right|}^{\infty} d x x^{4} e^{-x^{2}} \frac{\kappa_{B} \kappa_{p s}}{\kappa_{B}+\kappa_{p s}}\left(1, x^{2}-5 / 2\right)$, where $\kappa_{p s}(x)=\left(\nu_{i} \epsilon^{2} / 2 \sqrt{\pi}\right) \int_{-1}^{1} d y\left(1-3 y^{2}\right)\left(\nu_{*} x / \nu_{i}\right) \times\left\{\left(y+U_{p m} / x\right)^{2}+\left(\nu_{*} \epsilon^{3 / 2} \nu_{T}(x) / \nu_{i} x\right)^{2}\right\}^{-1}, \nu_{*}=$ $\nu_{i} R q /\left(\epsilon^{3 / 2} v_{t i}\right), \nu_{T}=3 \nu_{D}(x)+\nu_{E}(x)$ is the anisotropy relaxation frequency, $\kappa_{B}(x)=1.46 \sqrt{\epsilon}$ $\nu_{D}(8 / 3 \sqrt{\pi})\left(1-3 U_{p m}^{2} / x^{2}\right) \mathbb{F}\left(1+U_{p m} / x^{2}\right)^{-3 / 2}, \mathbb{F}=\left(1-U_{p m}^{2} / x^{2}\right)+\left(\nu_{\|} / \nu_{D}\right) U_{p m}^{2} / x^{2}, \nu_{D}, \nu_{E}$, and $\nu_{\|}$are defined in Ref. [24]. In the limit of $\left|U_{p m}\right| \rightarrow 0$, Eq. (6) reproduces standard viscosity coefficients that connect smoothly all collisionality regimes.

The nonlinearity in Eqs. (2) and (3) is in nonlinear viscosity shown in Eq. (6). Because of this nonlinearity, $U_{p m}$ can have bifurcated solutions. To show this, for simplicity, we 
assume particle source $S_{n a}$ vanishes. The momentum source can be eliminated by assuming $\left\langle\boldsymbol{B} \cdot \sum_{a} \boldsymbol{S}_{m a}\right\rangle \approx\left\langle\boldsymbol{B}_{t} \cdot \sum_{a} \boldsymbol{S}_{m a}\right\rangle$ and subtracting Eq. (3) from Eq. (2) to obtain

$$
\left\langle\boldsymbol{B}_{p} \cdot \nabla \cdot \sum_{a} \boldsymbol{\pi}_{a}\right\rangle=0,
$$

which is basically the poloidal momentum balance equation. Because $\left\langle\boldsymbol{B} \cdot \boldsymbol{\nabla} \cdot \boldsymbol{\pi}_{a}\right\rangle_{n}=$ $\left\langle\boldsymbol{B}_{p} \cdot \boldsymbol{\nabla} \cdot \boldsymbol{\pi}_{a}\right\rangle_{n}$ in Hamada coordinates, the explicity expression for Eq. (7) is $\left\langle\boldsymbol{B}_{p} \cdot \boldsymbol{\nabla} \cdot \sum_{a} \boldsymbol{\pi}_{a}\right\rangle_{n}+\left\langle\boldsymbol{B}_{p} \cdot \boldsymbol{\nabla} \cdot \sum_{a} \boldsymbol{\pi}_{a}\right\rangle_{1 / \nu}=0$. Substituting Eqs. (5) and (6) into (7), we obtain a dimensionless bifurcation equation

$$
\begin{aligned}
& \frac{M_{i} \mu_{1 i}}{M_{e} \mu_{1 e}}\left(U_{p m}-\bar{A}\right)=-U_{p m}-\bar{B} \\
& \quad-\frac{1}{N q} \frac{32 G(\alpha)}{9(2 \pi)^{3 / 2}} \frac{1}{\nu_{* e} \nu_{* \delta}}\left(\frac{\nu_{e} \sqrt{\epsilon}}{\mu_{1 e}}\right) 27.4\left(U_{p m}-\frac{V_{\| i}}{v_{t i}}-\bar{C}\right),
\end{aligned}
$$

where $\nu_{* e}=\nu_{e} R q /\left(v_{t e} \epsilon^{3 / 2}\right), \quad \nu_{e}$ is the electron-electron collision frequency, $\nu_{* \delta}=$ $\nu_{i} R /\left(v_{t i} N \delta^{3 / 2}\right), \bar{A}=-\left[c /\left(e v_{t i} B_{p}\right)\right]\left(p_{i}^{\prime} / n_{i}+\mu_{2 i} T_{i}^{\prime} / \mu_{1 i}\right), \bar{B}=-\left[c /\left(e v_{t i} B_{p}\right)\right]\left(p_{e}^{\prime} / n_{i}+\mu_{2 e} T_{e}^{\prime} / \mu_{1 e}\right)$, and $\bar{C}=-\left[c /\left(e B_{p} v_{t i}\right)\right]\left(p_{i}^{\prime} / n_{i}+2.37 T_{i}^{\prime}\right)$. Parallel heat flows are ignored in Eq. (8) because heat frictions are larger than heat viscosities in a large aspect ratio tokamak and to the leading order $q_{\| i} \approx q_{\| e} \approx 0$. Note that $\left(V_{\| i} / v_{t i}\right)$ in Eq. (8) denotes the coupling between Eq. (8) and Eq. (3). However, because $\left|U_{p m}\right| \sim 1$ and $\left|V_{\| i} / v_{t i}\right|<1$, we ignore $\left(V_{\| i} / v_{t i}\right)$ in Eq. (8) and thus decouple Eqs. (3) and (8).

Equation (8) is a nonlinear equation for $U_{p m}$. The solution is found graphically by plotting the right side and left side of the equation versus $U_{p m}$ as shown in Figs. 1-3. The parameters used are $n_{e}=3 \times 10^{19} \mathrm{~cm}^{-3}, \bar{A}=\bar{B}=0.2, T_{e}=5.5 \mathrm{keV}, B=4.6 \mathrm{~T}, R=2.6 \mathrm{~m}$, $a=0.8 \mathrm{~m}, \epsilon=0.1, N=20, q=2.5, \delta=1.3 \times 10^{-3}$, and $V_{\| i} / v_{t i}=0$. In Fig. 1, for $T_{i}=5.5 \mathrm{keV}$ and $\bar{C}=0.8$, there is only one solution and $U_{p m}<1$. This is the conventional solution. As $\bar{C}$ and $T_{i}$ increase to $\bar{C}=2.75$ and $T_{i}=6.5 \mathrm{keV}$, there can be three solutions as shown in Fig. 2. One of the new solutions, the one in the middle, is unstable. The other stable new solution is in the $U_{p m}>1$ regime. When $\bar{C}$ and $T_{i}$ increase further to $\bar{C}=3.0$ and $T_{i}=7.5 \mathrm{keV}$, the conventional solution disappears and only the stable $U_{p m}>1$ solution exists. Note that the most effective way to increase $\bar{C}$ is to increase ion temperature gradient 
because the numerical coefficient in front of $d T_{i} / d r$ is larger than that of $d n_{e} / d r$. When the heating power increases, both $T_{i}$ and $\bar{C}$ increase. As $T_{i}$ and $\bar{C}$ increase, $U_{p m}$ increases and eventually bifurcates from $U_{p m}<1$ to $U_{p m}>1$ regime. This sudden increase of $U_{p m}$ is similar to the observed abrupt jump of $E_{r}$ in ERS mode in TFTR. The bifurcated value of $U_{p m}$ is about 2 in this example. The observed $U_{p m}$ is greater than unity [11].

After bifurcation, turbulence fluctuation level is reduced by a factor of $1 /\left[1+\kappa\left(\omega^{\prime}\right)^{2}\right]$, where $\omega^{\prime}$ is the radial gradient of the $\boldsymbol{E} \times \boldsymbol{B}$ and diamagnetic angular velocity, and $\kappa$ is a normalization constant, and plasma confinement improves [25]- [27].

The fact that magnetic shear is reversed is not employed explicitly in the theory. It could be that reversed magnetic shear stabilizes magnetohydrodynamic (MHD) activities, such as sawteeth, and therefore reduces magnetic stress associated with the perturbed $\boldsymbol{J} \times \boldsymbol{B}$ force. Magnetic stress increases non-ambipolar electron loss and may prevent bifurcation of $U_{p m}$. It could also be that turbulence fluctuation levels are lower inside the $q_{\min }$ radius [28], therefore it is easier to increase $T_{i}$ and $d T_{i} / d r$ with heating power inside $q_{\min }$ radius, which in turn facilitate the bifurcation. Here $q_{\min }$ is the minimum value of $q$.

The plasma parameters in Figs. 1-3 are at the tail-end of $1 / \nu$ regime. If $T_{i}$ increase further, ripple trapping viscosity will move into the $\nu$ regime [14] and its magnitude will decrease as $\nu$ decreases. This is the likely cause for the ultimate relaxation of $U_{p m}$. The other possible cause is the magnetic stress associated with the MHD activity observed after bifurcation. The effect of neutral change exchange momentum loss [29] on the value of $U_{p m}$ can be important because for high temperature plasmas, nonlinear viscosity is weak. These issues will be addressed separately.

In summary, we develop a theory for enhanced reversed shear mode [30]. The physical process is as follows: (1) Reversed magnetic shear stabilizes MHD activities which reduces magnetic stress and reduces the turbulence fluctuations inside $q_{\min }$ radius; (2) When the heating power increases, both $T_{i}$ and $d T_{i} / d r$ increase. This leads to bifurcation of $U_{p m}$; (3) After $U_{p m}$ bifurcation, turbulence fluctuation level reduces by a factor of $1 /\left[1+\kappa\left(\omega^{\prime}\right)^{2}\right]$;

(4) Evolution of the plasma profiles relaxes $U_{p m}$; (5) However, $\left(\omega^{\prime}\right)^{2}$ is still substantial to 
suppress turbulence fluctuations and improve plasma confinement.

\section{Acknowledgments}

The authors would like to thank R.E. Bell for many stimulating discussions. This work was supported by the U.S. Dept. of Energy Contract No. DE-FG03-96ER-54346. 


\section{REFERENCES}

[1] ASDEX Team, Nucl. Fusion 29, 1959 (1989).

[2] R.J. Taylor, M.L. Brown, B.D. Fried, H. Grote, J.R. Liberati, G.J. Morales, and P. Pribyl, Phys. Rev. Lett. 63, 2365 (1989).

[3] R.J. Groebner et al., Phys. Rev. Lett. 64, 3015 (1990).

[4] K. Ida et al., Phys. Rev. Lett. 65, 1364 (1990).

[5] F. Wagner et al., Plasma Phys. Control. Fusion 36, A61 (1994).

[6] K. Toi et al., in Plasma Physics and Controlled Nuclear Fusion Research, 1994 (International Atomic Energy Agency, Vienna, 1994), Vol. 2, p. 331.

[7] K.C. Shaing and E.C. Crume, Jr., Phys. Rev. Lett. 63, 2369 (1989).

[8] S.-I. Itoh and K. Itoh, Phys. Rev. Lett. 60, 2376 (1989).

[9] F.M. Levinton, M.C. Zarnstorff, S.H. Bartha, M. Bell, R.E. Bell, R.V. Budny, C.E. Bush, Z. Chang, E.D. Fredrickson, A. Janos, J. Manickam, S.T. Ramsey, G.L. Schmidt, E.J. Synakowski, and G. Taylor, Phys. Rev. Lett. 75, 4417 (1995).

[10] S. Ishida et al., Phys. Rev. Lett. 79, 3917 (1997).

[11] R.E. Bell, Bull. Am. Phys. Soc. 42, 1945 (1997) (to be submitted to Phys. Rev. Lett. ) .

[12] K. Itoh and S.-I. Itoh, Nucl. Fusion 32, 2243 (1992).

[13] K.C. Shaing, Phys. Rev. Lett. 76, 4364 (1996).

[14] P.N. Yushmanov, Nucl. Fusion 22, 315 (1982).

[15] J.W. Connor and R.J. Hastie, Nucl. Fusion 13, 221 (1973).

[16] A.H. Boozer, Phys. Fluids 23, 2282 (1980). 
[17] R.J. Goldston, R.B. White, and A.H. Boozer, Phys. Rev. Lett. 47, 647 (1981).

[18] S. Hamada, Prog. Theo. Phys. (Kyoto) 22, 145 (1959).

[19] K.C. Shaing, S.P. Hirshman, and J.D. Callen, Phys. Fluids 29, 521 (1986); The reason ripple plateau flux is shown here is to illustrate the two-dimensional nature of the momentum equation to be solved.

[20] M.N. Rosenbluth et al. in Plasma Physics and Controlled Nuclear Fusion Research, 1970 (International Atomic Energy Agency, Vienna, 1971), Vol. 1, p. 495.

[21] T.E. Stringer, Nucl. Fusion 16, 12 (1972).

[22] K.C. Shaing, C.T. Hsu, and N. Dominguez, Phys. Plasmas 1, 1168 (1994).

[23] K.C. Shaing, Phys. Fluids B 2, 2847 (1990).

[24] S.P. Hirshman and D.J. Sigmar, Nucl. Fusion 21, 1079 (1981).

[25] K.C. Shaing et al., in Plasma Physics and Controlled Nuclear Fusion Research, 1988 (International Atomic Energy Agency, Vienna, 1989), Vol. 2, p. 13; K.C. Shaing, E.C. Crume, Jr., and W.A. Houlberg, Phys. Fluids B 2, 1496 (1990).

[26] K. Itoh et al., Plasma Phys. Control. Fusion 36, 123 (1994).

[27] Y.Z. Zhang and S.M. Mahajan, Phys. Fluids B 4, 1385 (1992).

[28] K. Kishimoto, et al., in Fusion Energy, (International Atomic Energy Agency, Vienna, 1996), vol. 2, p. 581.

[29] M. Coronado and J.N. Talmadge, Phys. Fluids B 5, 1200 (1993).

[30] Whether this theory is applicable to Negative Central Shear (NCS) mode observed in DIII-D [E.J. Strait, et al., Phys. Rev. Lett. 75, 4421 (1995)] remains to be seen. 


\section{FIGURE CAPTIONS}

FIG. 1. Right side (RHS) and left side (LHS) of Eq. (8) versus $U_{p m}$. There is one solution which corresponds to the conventional confinement mode.

FIG. 2. As $T_{i}$ and $\bar{C}$ increase, the value of $U_{p m}$ increases. Here, there are three solutions. The one in the middle is unstable. The solution with the longest value of $U_{p m}$ is the new stable solution, which corresponds to the ERS mode.

FIG. 3. When $T_{i}$ and $\bar{C}$ increase further, only the ERS mode solution exists. 